\title{
Phason Contribution to the Dislocation Loop Bias in Quasicrystals
}

\author{
G.N. Lavrova*, A.A. Turkin And A.S. Bakai
}

NSC Kharkov Institute of Physics and Technology, Kharkov 61108, Ukraine

\begin{abstract}
We develop a model of the absorption of vacancies and self-interstitial atoms by dislocation loops and associated phason defects in quasicrystals under irradiation. The capture efficiency and the bias of the loop for radiation point defects are evaluated for variable loop sizes. Numerical calculation of these quantities is performed for comparison. It is shown that phason defects decrease the total bias of the dislocation loop in a quasicrystal.
\end{abstract}

DOI: 10.12693 /APhysPolA.126.505

PACS: 61.44.Br, 61.72.Lk, 61.80.Az

\section{Introduction}

Physical phenomena in structural materials caused by irradiation are referred to as radiation damage [1]. Bombardment of solids by fast particles leads to the formation of radiation point defects - the Frenkel pairs (vacancies and self-interstitials) in the bulk. Extended defects such as grain boundaries, dislocations, voids, etc. act as the sinks for these point defects. The separation of fluxes of vacancies and self-interstitial atoms between different sinks induces the majority of radiation effects. The main mechanism for the flux separation is the dislocation bias arising due to the strong elastic interaction of self-interstitials with dislocations.

The influence of irradiation on quasicrystals has not been studied extensively yet. However due to their specific structure one can assume that their behavior under irradiation would differ from that of periodic crystals. Here we calculate the absorbing capacity of dislocation loops in quasicrystals and compare it with that in periodic crystals.

\section{Analytical model}

In a periodic crystal a growing dislocation loop encloses a perfect crystal. Thus the loop absorbs radiation point defects only on the dislocation line itself. Unlike periodic crystals, quasicrystals lack translational invariance. Hence shifting two parts of a quasicrystal leads to mismatches of the quasicrystalline lattice loosely called phasons. It has been experimentally observed that a dislocation moving in a quasicrystal trails a layer of phason defects in its wake [2]. The phason trail forms a phason wall that disperses in the bulk with increasing temperature. In view of this, we consider a simplified model of a circular dislocation loop under irradiation containing a phason "disc" inside which acts as a neutral sink. The temperature is presumed to be sufficiently low for the phason disc to be stable in the bulk.

*corresponding author; e-mail: g. lavrova@kipt.kharkov.ua
Brailsford and Bullough [3] developed a theory of dislocation loop sink strength and bias in crystals. In order to be applicable for quasicrystals this model has to be modified taking into account the phason fault of the dislocation loop.

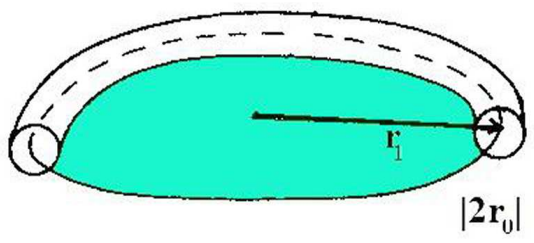

Fig. 1. Dislocation loop with the phason trail inside.

A toroidal dislocation loop of radius $r_{1}$ with the phason disc-like defect (Fig. 1) is to be placed in the effective medium. Neglecting the generation of point defects and other sinks, a simplified diffusion problem for the point defect concentration $C$ near the dislocation loop is

$$
\nabla^{2} C=0,
$$

where $\nabla^{2}$ is the Laplacian, subject to the conditions

$$
\begin{aligned}
& C(R)=\bar{C}, \\
& \left.\frac{\partial C}{\partial n}\right|_{S_{s}}=\frac{C_{1} v_{n}}{D},
\end{aligned}
$$

where $R$ is the size of the sample, $\frac{\partial}{\partial n}$ is a normal derivative taken in the direction normal to the sink surface of the loop and phason disc $S_{s}, v_{n}$ is the transfer velocity on the sink surface $S_{s}, C_{1}$ is the concentration there, $C_{1} v_{n}$ is the point defect flux density at the loop surface, $D$ is the diffusion coefficient. We use the electrostatic analogy to this problem [4] to calculate the capture efficiency of the sink for point defects, $Z^{\mathrm{v}, \mathrm{i}}$ :

$$
Z^{\mathrm{v}, \mathrm{i}}=\frac{J}{2 \pi r_{1} D \bar{C}},
$$

where $J$ is the total point defect flux on the sink surface. The concentration $C$ is considered as the counterpart of the electrical potential and $\partial C / \partial n$ as the counterpart of the magnitude of the electric field at the surface of a metal torus and metal disc. 
Thus the diffusion problem for quasicrystals can be solved analytically in the approximation of a simple superposition of sinks. Evidently, the interference of the effects of sinks in this approximation is ignored. In this case the capture efficiencies of a dislocation loop for vacancies and self-interstitials are

$$
Z_{\mathrm{qcr}}^{\mathrm{v}, \mathrm{i}}=\frac{2 \pi}{\ln \left(\frac{8 r_{1}}{r_{0}^{\mathrm{v}, \mathrm{i}}}\right)}+\frac{4}{\pi},
$$

where $r_{0}^{\mathrm{v}}, r_{0}^{\mathrm{i}}$ are the point defect capture radii for vacancies and self-interstitials, respectively.

The bias factor determines the swelling rate of a material under irradiation. The dislocation bias is defined as follows:

$$
B_{\mathrm{qcr}}=1-\frac{Z_{\mathrm{qcr}}^{\mathrm{v}}}{Z_{\mathrm{qcr}}^{\mathrm{i}}} \text {. }
$$

The dependences of the dislocation loop capture efficiencies and the loop bias on loop radius (units of the Burgers vector length $b$ ) for periodic crystals and quasicrystals are calculated using experimentally obtained parameters for the Al-Pd-Mn quasicrystal (see Figs. 2 and 3 ). The capture radius for vacancies is considered to be $r_{0 \mathrm{v}}=b$, and for the self-interstitials: $r_{0 \mathrm{i}}=3 b$.



Fig. 2. Interstitial and vacancy capture efficiencies of the dislocation loop in a periodic crystal $\left(Z^{\mathrm{i}}, Z^{\mathrm{v}}\right)$ and a quasicrystal $\left(Z_{\mathrm{qcr}}^{\mathrm{i}}, Z_{\mathrm{qcr}}^{\mathrm{v}}\right.$ ) as the functions of loop radius.

As expected, the capture efficiencies for point defects in a quasicrystal are greater than those in a periodic crystal because of the higher concentration of sinks. The bias of a dislocation loop towards self-interstitials, on the other hand, is lower in a quasicrystal, and it significantly depends on the loop radius.

\section{Numerical calculations}

The model we have used for our estimations of the dislocation loop capture efficiency and the bias factor is a very simplified one; therefore the results obtained are rather qualitative. Since currently there are no experimental data to compare our results with, we have used a numerical method to calculate the capture efficiencies of

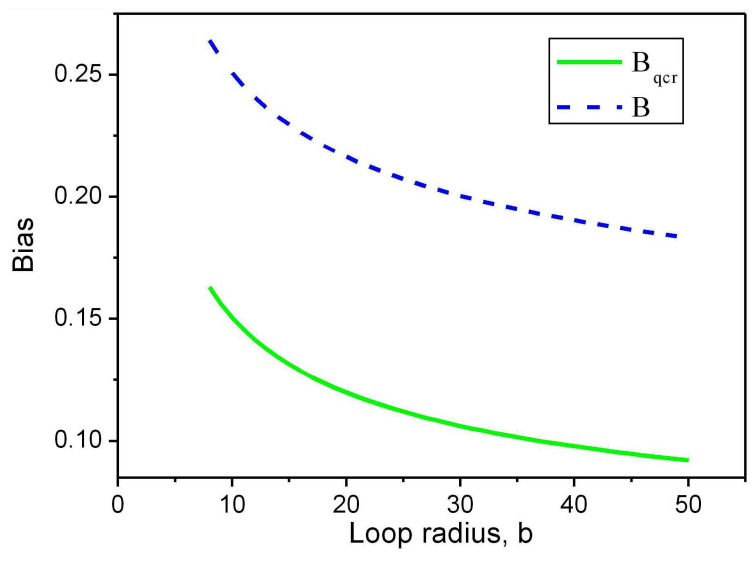

Fig. 3. Dislocation loop bias in a periodic crystal $(B)$ and a quasicrystal $\left(B_{\mathrm{qcr}}\right)$ as a function of loop radius.

dislocation loops in a quasicrystal. Similarly to Ref. [5] the problem of point defect diffusion is considered in a spherical sink-free region of radius $R>r_{1}$ enclosing the dislocation loop with the phason defect

$\operatorname{div} \boldsymbol{j}=0$.

The flux of point defects interacting with the stress field of the dislocation is given by

$$
\boldsymbol{j}=-D \nabla C-\frac{D C}{k_{\mathrm{B}} T} \nabla E,
$$

where $E$ is the interaction energy [6] of the dislocation loop with a point defect that is modeled by a dilation center.

The boundary condition at the external boundary of the sink-free region is given by Eq. (2). At the surface of the phason defect and at the dislocation core the point defect concentration is assumed to be zero (absorbing boundary condition).

The differential Eq. (7) and the associated boundary conditions are transformed into a set of finite difference equations, which are then solved by the modified Liebmann method [7] to find the total flux $J$. The capture efficiency is defined by Eq. (4).

Calculations were performed for icosahedral quasicrystalline $\mathrm{Al}_{72} \mathrm{Pd}_{20} \mathrm{Mn}_{8}$ at $T=1000 \mathrm{~K}$ using experimentally obtained material parameters [2, 8-10]. The dilatation volumes of vacancies and self-interstials are assumed to be $\Omega_{\mathrm{v}}=-0.5 \omega$ and $\Omega_{\mathrm{i}}=\omega$, respectively (here $\omega$ is the mean atomic volume). The results of numerical calculations are presented in Figs. 4 and 5.

These figures show that the capture efficiency does not depend on the type of the material for small loop sizes. That is due to the fact that the effective radius of elastic interaction with the point defect is greater than the dislocation loop radius for small loops. In other words such loops behave as three-dimensional sinks whose internal structure does not affect the total point defect flux. 


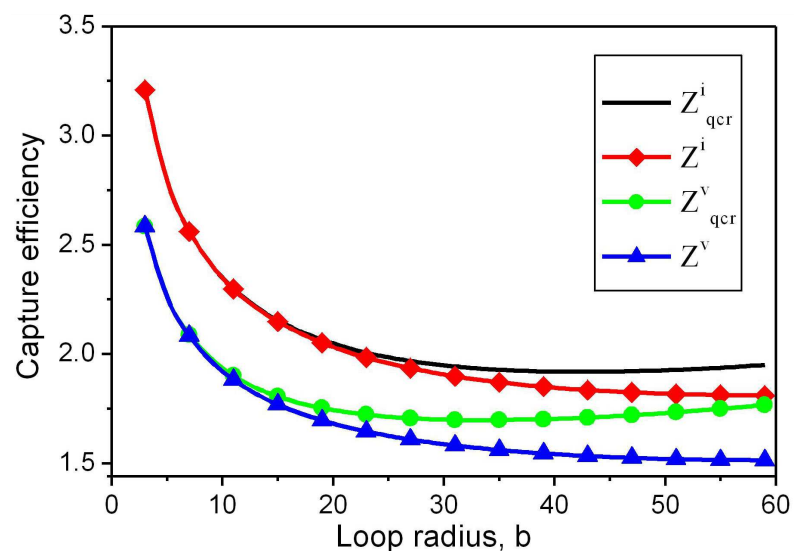

Fig. 4. Numerical calculation of the interstitial and vacancy capture efficiencies of the dislocation loop in a periodic crystal $\left(Z^{\mathrm{i}}, Z^{\mathrm{v}}\right)$ and a quasicrystal $\left(Z_{\mathrm{qcr}}^{\mathrm{i}}, Z_{\mathrm{qcr}}^{\mathrm{v}}\right)$ as the functions of loop radius.

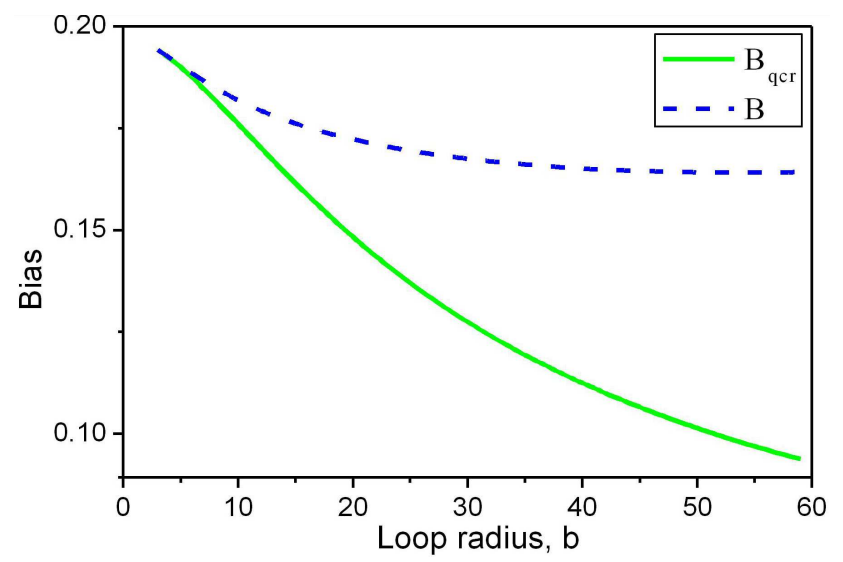

Fig. 5. Numerical calculation of the dislocation loop bias in a periodic crystal $(B)$ and a quasicrystal $\left(B_{\mathrm{qcr}}\right)$ as a function of loop radius.

\section{Conclusions}

We have put forward a model of a dislocation loop enclosing a non-dispersing phason trail subjected to irradiation. We have found that the capture efficiency of the dislocation loop for point defects in a quasicrystal is greater than that in a periodic crystal due to absorption of point defects by phasons. The bias of the dislocation loop in a quasicrystal is lower than that in a periodic crystal with the same material parameters because of the contribution of phason defects. The difference in dislocation loop bias for periodic crystals and quasicrystals increases with the loop radius. We performed numerical calculations to support the analytical model. Quasicrystals are expected to be more resistant to swelling under irradiation.

\section{References}

[1] G.S. Was, Fundamentals of Radiation Materials Science, Springer-Verlag, Berlin 2007.

[2] F. Mompiou, D. Caillard, M. Feuerbacher, Philos. Mag. 84, 2777 (2004).

[3] A.D. Brailsford, R. Bullough, Philos. Trans. R. Soc. Lond. 302, 87 (1981).

[4] G.N. Lazareva, A.S. Bakai, J. Kharkiv Natl. Univ., Phys. Series 1041, (2/58) 64 (2013).

[5] V.I. Dubinko, A.S. Abysov, A.A. Turkin, J. Nucl. Mater. 336, 11 (2005).

[6] J. Baštecká, F. Kroupa, Czech. J. Phys. B 14, 443 (1964).

[7] G.D. Smith, Numerical Solution of Partial Differential Equations, Oxford University Press, London 1965.

[8] M. Feuerbacher, M. Weller, J. Diehl, K. Urban, Philos. Mag. Lett. 74, 81 (1996).

[9] R. Blüher, P. Scharwaechter, W. Frank, H. Kronmüller, Phys. Rev. Lett. 80, 1014 (1998).

[10] J.T. Okada, M. Inui, D. Ishikawa, A.Q.R. Baron, K. Matsuda, S. Tsutsui, Y. Watanabe, J. Phys., Condens. Matter 18, L613 (2006). 